\title{
RESISTÊNCIA ANAERÓBICA \\ DE JOGADORES DE FUTEBOL
}

\author{
Edyta Sienkiewicz-Dianzenza' edyła.sienkiewicz@awf.edu.pl \\ Marcin Rusin² rusek_86@interia.eu \\ Romuald Stupnicki² rstupnicki@poczta.onet.pl
}

doi:10.3900/fpi.8.3.199.p

Sienkiewicz-Dianzenza E, Rusin M, Stupnicki R. Resistência anaeróbica de jogadores de futebol. Fit Perf J. 2009 mai-jun;8(3):199-203.

\section{RESUMO}

Introdução: A resistência anaeróbica, ou seja, a capacidade de realizar séries repetidas de exercícios anaeróbicos na potência máxima é uma característica indispensável para os jogadores de futebol. $\bigcirc$ objetivo do estudo foi determinar a resistência anaeróbica de jogadores de futebol aplicando repetidos sprints em velocidade máxima. Materiais e Métodos: Um grupo de 14 jogadores de futebol com idades entre 19 e 27 anos foi submetido duas vezes, em janeiro e março, a um teste consistindo de corridas máximas de $6 \times 50 \mathrm{~m}$ com $15 \mathrm{~s}$ de intervalo entre as corridas. Os tempos registrados foram convertidos para velocidades. A proporção da velocidade média para a máxima, obtida em todas as seis corridas, ou índice de performance (IP), foi a medida da resistência anaeróbica.

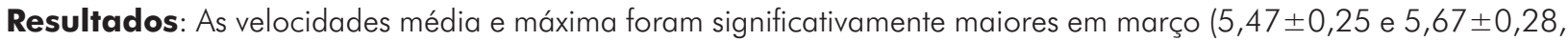

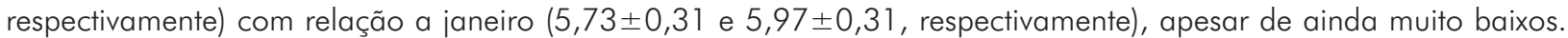
Os valores de IP foram similares nas duas ocasiões $(0,960$ e 0,965) e não foram correlacionados com as velocidades máximas. Discussão: $\bigcirc$ método apresentado pode servir como uma ferramenta útil para determinar e classificar atletas no que diz respeito a suas resistências anaeróbicas, aplicando séries repetidas de um exercício específico.

\section{PALAVRAS-CHAVE}

Resistência Física, Desempenho Atlético, Futebol.

\footnotetext{
1 University of Physical Education - Warsaw - Polônia

${ }^{2}$ Rzeszów University - Department of Physical Education - Rzeszów - Polônia
}

Copyright(C 2009 por Colégio Brasileiro de Atividade Física, Saúde e Esporte

Fit Perf J | Rio de Janeiro | 8 | 3 | 199-203 | mai/jun 2009 


\section{Anaerobic resistance of soccer players}

\section{ABSTRACT}

Introduction: Anaerobic resistance, i.e. the capacity to perform repeated series of anaerobic exercises at the maximum, is an indispensable feature for soccer players. The aim of the study was thus to determine the anaerobic resistance of soccer players by applying repeated maximal sprints. Materials and Methods: A group of 14 soccer players aged $19-27$ years-old was subjected twice, in January and March, to a test consisting of maximal running 6'50 m with 15-s intermissions between runs. The registered times were converted to speeds. The ratio of average speed to the maximum one achieved in all 6 runs, or the Performance Index (PI), was the measure of anaerobic resistance. Results: Average and maximum speeds were significantly $(p<0.001)$ higher in March $(5.47 \pm 0.25$ and $5.67 \pm 0.28$, respectively) than in January $(5.73 \pm 0.31$ and $5.97 \pm 0.31$, respectively), although still rather low. The PI values were on both occasions alike (0.960 and 0.965$)$ and were not correlated with maximum speeds. Discussion: The presented method may serve as a useful tool in determining and classifying athletes with respect to their anaerobic resistance by applying repeated series of specific exercise.

\section{KEYWORDS}

Physical Endurance, Athletic Performance, Soccer.

\section{Resistencia ANAERÓbICA DE LOS JUgAdORES DE FÚtBOL \\ RESUMEN}

Introducción: La resistencia anaeróbica, es decir, la capacidad de realizar series repetidas de ejercicios anaeróbicos en la potencia máxima es una característica indispensable para los jugadores de fútbol. El objetivo del estudio fue a determinar la resistencia anaeróbica de jugadores de fútbol aplicando repetidos sprints en velocidad máxima. Materiales y Métodos: Un grupo de 14 jugadores de fútbol con edades entre 19 y 27 años fue sometido dos veces, en enero y marzo, a un test consistiendo de carreras máximas de $6 \times 50 \mathrm{~m}$ con 15s de intervalo entre las carreras. Los tiempos registrados habían sido convertidos para velocidades. La proporción de la velocidad media para la máxima, obtenida en todas las seis carreras, o índice de performance (IP), fue la medida de la resistencia anaeróbica. Resultados: Las velocidades media y máxima fueron significativamente mayores en marzo $(5,47 \pm 0,25$ y $5,67 \pm 0,28$, respectivamente) con respecto a enero $(5,73 \pm 0,31$ y $5,97 \pm 0,31$, respectivamente), a pesar de todavía mucho bajos. Los valores de IP fueron similares en las dos ocasiones $(0,960$ y 0,965$)$ y no habían sido correlacionados con las velocidades máximas. Discusión: El método presentado puede servir como una herramienta útil para determinar y clasificar atletas por lo que respecta a suyas resistencias anaeróbicas, aplicando series repetidas de un ejercicio específico.

\section{PALABRAS CLAVE}

Resistencia Física, Rendimiento Atlético, Fútbol.

\section{INTRODUÇÃO}

Resistência anaeróbica (RA), definida como a capacidade de realizar uma tarefa consistindo de séries repetidas de exercício anaeróbico na potência máxima ${ }^{1}$, é uma característica indispensável para jogadores de futebol. A definição de RA é, assim, próxima à resistência de sprint ${ }^{2}$, ou seja, a capacidade de realizar vários sprints e/ou séries de explosão, sem perda substancial de velocidade. De acordo com MacDougall et al. ${ }^{3}$ e Vrabas et al. ${ }^{4}$, essa capacidade é relacionada à adaptação, induzida por treinamento em alta altitude, para um uso eficiente de oxigênio pelas células musculares. Assim, jogadores de futebol possuindo alta resistência anaeróbica podem exibir uma alta resistência à fadiga e rápida recuperação após seguidos exercícios repetidos realizados durante uma partida, como chutes, sprints e outras ações. Quando os intervalos entre estes exercícios não são longos o bastante, a ressíntese das fontes de fosfágeno é insuficiente e a energia seria suprida pela glicose láctica, levando à fadiga e diminuição do performance ${ }^{5}$.

Nos estudos encontrados envolvendo exercícios repetidos de curta duraçãa ${ }^{6,7,8,9,10,11,12,13,14,15}$ os autores analisaram exercícios individuais em séries, mas não o exercício inteiro consistindo de várias séries. Desta forma, o método previamente descrito usando o comumente denominado índice de performance $(I P)^{16,17,18,19,20}$ foi usado neste estudo para determinar a resistência anaeróbica de jogadores de futebol.

\section{Objetivo}

O objetivo do estudo foi determinar os efeitos de um regime de treinamentos padrão no período de treinamento 
preliminar, na resistência anaeróbica de jogadores de futebol avaliados através da aplicação de sprints máximos repetidos.

\section{MATERIAIS E MÉTODOS}

Um grupo de 14 jogadores de futebol do clube "Glogovia", com idades entre 19 e 27 anos, se voluntariaram a participar do estudo. $\bigcirc$ grupo incluía dois goleiros, dois atacantes, cinco zagueiros e cinco meio-campistas. As suas alturas variaram entre $162 \mathrm{~cm}$ e $188 \mathrm{~cm}$, massa corporal entre $65 \mathrm{~kg}$ e $88 \mathrm{~kg}$, e experiência em treinamentos de 6 a 14 anos.

Todos os jogadores foram submetidos a testes de máxima corrida consistindo de seis sprints, de 50m cada, separados por $15 \mathrm{~s}$ de intervalos passivos. Os tempos de cada sprint foram registrados e convertidos para velocidades, a fim de produzir valores decrescentes. $\bigcirc$ teste foi precedido de um aquecimento durante cerca de 10min, e consistiu de exercícios dinâmicos e um sprint único de $50 \mathrm{~m}$ na velocidade máxima. As medidas foram realizadas duas vezes: no início (janeiro) e no final (março) do treinamento preliminar para o segundo turno do campeonato.

O RA, ou seja, a capacidade de manter ao máximo a velocidade de corrida durante o teste, foi expressa como o $\mid P^{16,17,18,19,20}$, definido como a proporção do valor médio registrado na série de exercícios na máxima potência no teste. As frequências cardíacas foram medidas manualmente antes do teste (mas após o aquecimento) e após o teste.

A ANOVA Three-way (sujeitos - series de exercícios períodos) foi aplicada aos dados de velocidade seguidos pelo teste Post-Hoc de Scheffé para os dados pareados e o teste " $t$ " de Student para a diferença nos valores de IP. As relações entre os resultados pré e pós-treino ou entre as velocidades e os valores de IP foram determinados pelos coeficientes de correlação de Pearson. $\bigcirc$ nível de $\mathrm{p} \leq 0,05$ foi considerado significativo.

\section{RESULTADOS}

Os valores médios ( \pm desvio padrão) registrados no grupo estudado, tanto os somáticos como os relacionados à performance, estão presentes na Tabela 1 . As velocidades média e máxima alcançadas em março foram significativamente mais altas do que em janeiro $(p<0,001)$. Em contraste, as frequências cardíacas médias, tanto pré quanto pós-exercício, foram significativamente mais baixas $(p<0,01)$. Não foi observada diferença significativa quanto ao IP.

As velocidades médias de corrida ( \pm desvio padrão) alcançadas em seis sprints consecutivos, em dois períodos de exames, estão mostrados na Figura 1. As rotas são não-lineares, especialmente em março. As velocidades mais altas foram alcançadas no segundo ou terceiro sprint e variaram entre $5,60 \pm 0,30$ e 5,82 $\pm 0,29 \mathrm{~m} \cdot \mathrm{s}^{-1}$ (médias \pm desvio padrão) em janeiro e março, respectivamente, com valores individuais variando de $5,24 \mathrm{~m} . \mathrm{s}^{-1}$ a $6,05 \mathrm{~m} . \mathrm{s}^{-1}$ e de $5,36 \mathrm{~m} \cdot \mathrm{s}^{-1}$ a $6,25 \mathrm{~m} \cdot \mathrm{s}^{-1}$, respectivamente. A ANOVA revelou diferenças altamente significativas entre os sujeitos $\left(F_{13,65}=16,95 ; p<0,001\right)$ e entre os períodos dos exames $\left(F_{1,83}=109,3 ; p<0,001\right)$. Foi observada melhoria significativa $(p<0,001)$ nas velocidades de corrida em todos os sujeitos, exceto um zagueiro no qual nenhuma mudança significativa foi observada e o incremento de velocidade foi razoavelmente constante (Figura 2). Em contraste, os valores de IP registrados em ambas ocasiões não foram correlacionados (Figura 3).

A relação entre os valores do IP e o de velocidade máxima alcançados em séries de seis sprints está mostrado na Figura 4. A falta de correlação significativa $(r=-0,054)$ permitiu classificar os jogadores de acordo com a velocidade máxima de sprint e resistência anaeróbica. Entre os 14 jogadores estudados, dois (um atacante e um zagueiro) exibiram alta velocidade de sprint combinada com alta resistência anaeróbica.

Tabela 1 - Valores médios ( \pm desvio padrão) das variáveis registradas em jogadores de futebol $(\mathrm{n}=14)$

\begin{tabular}{|c|c|c|}
\hline variável & & \\
\hline idade (anos) & \multicolumn{2}{|c|}{$20,4 \pm 2,1$} \\
\hline altura corporal $(\mathrm{cm})$ & \multicolumn{2}{|c|}{$180,9 \pm 5,4$} \\
\hline \multirow[t]{2}{*}{ massa corporal $(\mathrm{kg})$} & \multicolumn{2}{|c|}{$73,4 \pm 7,4$} \\
\hline & janeiro & março \\
\hline velocidade média $\left(\mathrm{m} \cdot \mathrm{s}^{-1}\right)$ & $5,47 \pm 0,25$ & $5,73 \pm 0,31^{* *}$ \\
\hline velocidade máxima $\left(\mathrm{m} \cdot \mathrm{s}^{-1}\right)$ & $5,67 \pm 0,28$ & $5,97 \pm 0,31^{* *}$ \\
\hline índice de performance & $0,965 \pm 0,020$ & $0,960 \pm 0,018$ \\
\hline frequência cardíaca pré-exercício (bpm) & $108 \pm 10$ & $92 \pm 6^{*}$ \\
\hline frequência cardíaca pós-exercício (bpm) & $142 \pm 9$ & $126 \pm 11^{*}$ \\
\hline
\end{tabular}

significativamente diferente de seu respectivo valor em janeiro:

${ }^{*} p<0,01 ;{ }^{* *} p<0,001$ 


\section{DISCUSSÃO}

Os resultados alcançados por jogadores de futebol em março foram significativamente mais altos do que em janeiro, o que pode sugerir um efeito de treinamento ou menor engajamento dos sujeitos no teste de janeiro em relação do de março. $O$ engajamento e/ou a capacidade de corrida dos sujeitos foram insatisfatórios em todos os casos. A média de velocidade máxima obtida foi $5,97 \pm 0,31 \mathrm{~m} \cdot \mathrm{s}^{-1}$, marcadamente (por cerca de $1 \mathrm{~m} . \mathrm{s}^{-1}$ ) mais baixa do que a registrada num grupo representativo de esco-

Figura 1 - Valores médios ( \pm desvio padrão) da velocidade máxima de corrida alcançada por jogadores de futebol $(n=14)$ em uma série de sprints de $6 \times 50 \mathrm{~m}$, separados por intervalos de 15s, antes (janeiro) e após (março) o treinamento preliminar para o returno do campeonato

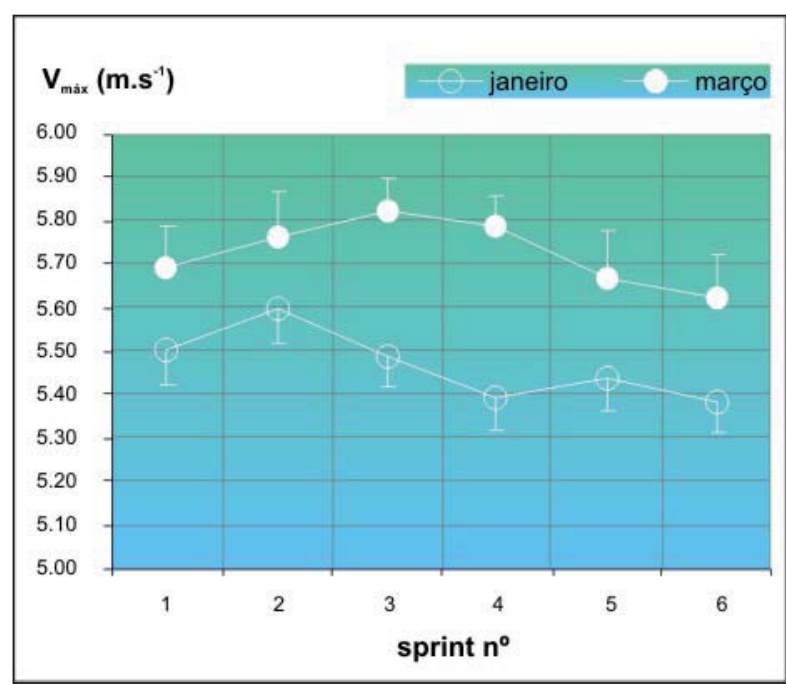

Figura 3 - Valores de índice de performance (IP) alcançados por indivíduos jogadores de futebol $(n=14)$, antes (janeiro) e após (março) o treinamento preliminar para o returno do campeonato

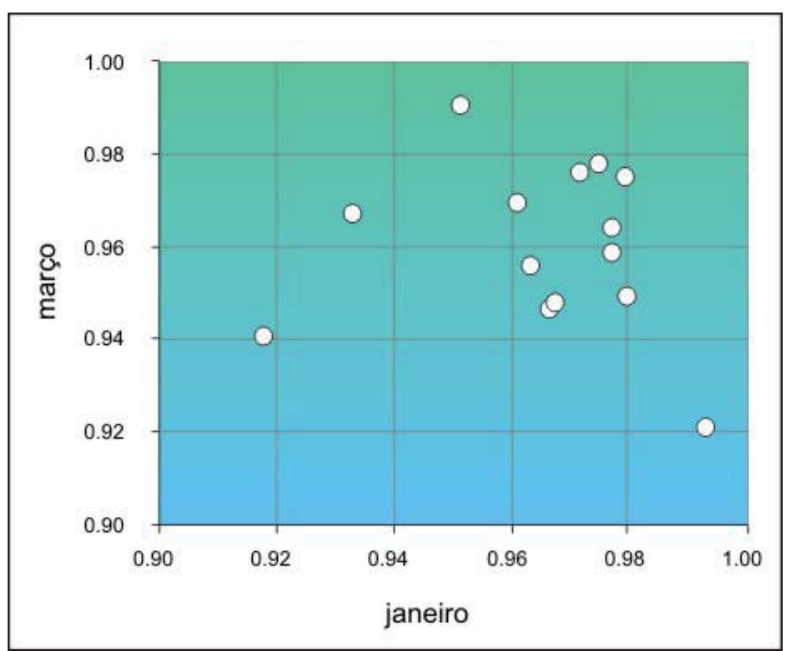

lares destreinados de 17 a 20 anos $^{20}$. Em contraste, os valores médios do IP medidos nas duas ocasiões não diferiram significativamente um do outro e foram muito altos - mais de 0,95 . Este IP alto foi outro sinal que os testes não foram realizados ao máximo. Caso contrário, a fadiga desenvolvida em corridas consecutivas seria mais notada.

O IP usado neste estudo se mostrou independente do número de séries num exercício ou das mudanças das $\operatorname{rotas}^{1,21}$. A falta de correlação entre o IP e a velocidade máxima, confirmada neste estudo, permitiu

Figura 2 - Velocidades máximas de sprint alcançadas por indivíduos jogadores de futebol $(n=14)$, antes (janeiro) e após (março) o treinamento preliminar para o returno do campeonato. A comumente chamada linha de identidade é mostrada.

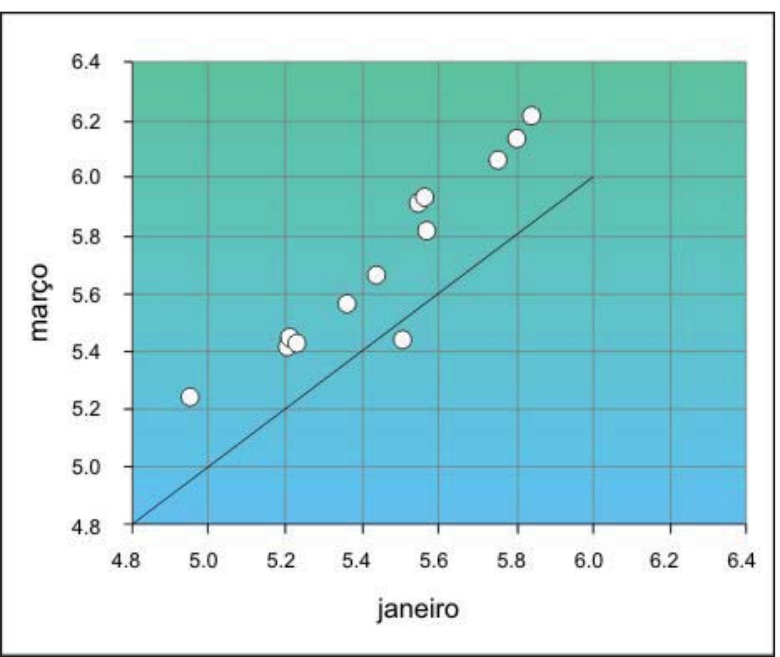

Figura 4 - Relação entre os valores do índice de performance (IP) e a velocidade máxima de sprint $\left(\mathbf{V}_{\text {max }}\right)$ alcançados por indivíduos jogadores de futebol $(n=14)$ após o treinamento preliminar para o returno do campeonato (março). O círculo, cujo raio é igual ao desvio padrão das duas variáveis, delimita a área da performance média.

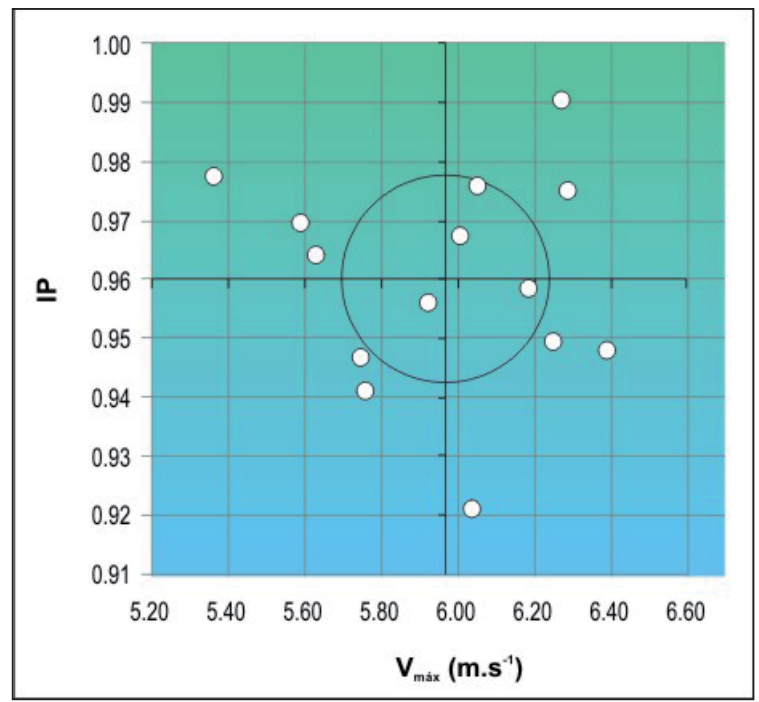


aplicar o gráfico de dispersão (Figura 4) para determinar e classificar os jogadores de futebol. A saber, os mais promissores seriam aqueles que conseguiriam alcançar a velocidade mais rápida de corrida e o mais alto $I P$, simultaneamente. Dois jogadores assim são mostrados no canto superior direito da Figura 4. Por outro lado, dois jogadores no canto inferior esquerdo são deficientes em ambos - velocidade e resistência anaeróbica.

Concluindo, o método utilizado neste estudo permite determinar jogadores individuais, no que diz respeito à sua resistência anaeróbica, ou seja, a capacidade de desempenhar uma tarefa consistindo de séries repetidas de exercício anaeróbico na potência máxima, determinando o $I P$, ou seja, a proporção entre a velocidade média e a máxima alcançadas em várias corridas máximas intercaladas por curtos intervalos. O IP não foi correlacionado com a velocidade máxima, permitindo, assim, classificar os jogadores e ajustar as cargas de treinamento às suas necessidades individuais.

\section{REFERÊNCIAS}

1. Stupnicki R, Sienkiewicz-Dianzenza E. Anaerobic resistance and its determinant. J Hum Kin. 2004;12:109-11.

2. Weineck J. Wie verbessere ich die Schnelligkeit. Fussballtraining. 1994;3:818.

3. MacDougall JD, Hicks AL, MacDonald JR, Mc Kelvie RS, Green HJ, Smith KM. Muscle performance and enzymatic adaptations to sprint interval training. J Appl Physiol. 1998;84:2138-42.

4. Vrabas IS, Dodd SL, Crawford MP. Interaction of blood flow and oxygen delivery affects peak $\mathrm{VO}_{2}$ and fatigue in canine muscle in situ. Eur J Appl Physiol. 2002;86:273-9.

5. Chmura J. Szybko w piłce no nei [Speed in soccer]. AWF Katowice; 2001.
6. Balsom PD, Gaitanos GC, Ekblom B, Siödin B. Reduced oxygen availability during high intensity intermittent exercise impairs performance. Acta Physiol Scand. 1994;152:279-85.

7. Balsom PD, Seger JY, Sjödin B, Ekblom B. Maximal-intensity intermittent exercise: effect of recovery duration. Int J Sports Med. 1992;13:528-33.

8. Balsom PD, Seger JY, Sjödin B, Ekblom B. Physiological responses to maximal intensity intermittent exercise. Eur J Appl Physiol. 1992;65:144-9.

9. Burgomaster KA, Hughes SC, Heigenhauser GJF, Bradwell SN, Gibala MJ. Six sessions of sprint interval training increases muscle oxidative potential and cycle resistance capacity in humans. J Appl Physiol. 2005;98:1985-90.

10. Gaitanos GC, Williams C, Boobis LH, Brooks S. Human muscle metabolism during intermittent maximal exercise. J Appl Physiol. 1993;75:712-9.

11. Greenhaff PI. Creatine and its application as an ergogenic aid. Intern J Sport Nutr. 1995;5:100-10.

12. Norkowski H, Kłossowski M, Bu ko K. Physiological responses to repeated series of anaerobic exercise. Phys Educ Sport 2002;46(Suppl. 1/1):576-8.

13. Odland LM, MacDougall JD, Tarnopolsky M, Elorriage A, Borgmann A, Atkinson $\mathrm{S}$. The effect of oral $\mathrm{Cr}$ supplementation of muscle ( $\mathrm{PCr}$ ) and power output during a short-term maximal cycling task. Med Sci Sports Exerc. 1994;26(Suppl 5):23.

14. Raastand T, Biøro T, Hallén J. Hormonal responses to high- and moderateintensity strength exercise. Eur J Appl Physiol. 2000;82:121-8.

15. Ratel S, Duche P, Hennegrave A, Van Praagh E, Bedu M. Acid-base balance during repeated cycling sprints in boys and men. J Appl Physiol. 2002;92:479-85.

16. Stupnicki R, Norkowski H. Index of anaerobic resistance in repeated maximal exercise series. Papers Anthropol. 2001;10:280-7.

17. Stupnicki $R$, Norkowski $H$, Hałda K. Resistance in repeated maximal exercise series. Medicina Sportiva 2000;1:E101.

18. Sienkiewicz E, Milde K, Dere M, Stupnicki R. An determinement of serially repeated arm exercises. Proc $3^{\text {rd }}$ Intern Conf. "Student, science and sport in the $21^{\text {st }}$ Century". Kyiv, Ukraine; 2002.

19. Sienkiewicz E, Milde K, Norkowski H, Stupnicki R. Determinement of repeated, high-intensity leg exercises. Wych Fiz Sport. 2002;46(Supll 1/1):437-8.

20. Stupnicki R, Dobosz J, Tomaszewski P, Milde K. Standardisation of selected somatic and physical fitness variables. Phys Educ Sport. 2005;49:72-9.

21. Sienkiewicz-Dianzenza E, Stupnicki R. Performance index - a better measure of repeated series of maximal exercise than linear regression. Proc $11^{\text {th }}$ Conf "Sport Kinetics 2003". Rydzyna; 2003.

Recebido: 13/11/08 - Aceito: 22/02/09 